\title{
PROGNOSE DO CRESCIMENTO E DA PRODUÇÃO EM CLASSES DE DIÂMETRO PARA POVOAMENTOS DESBASTADOS DE Eucalyptus grandis NO SUL DO BRASIL ${ }^{1}$
}

\author{
Fabiane Aparecida de Souza Retslaff ${ }^{2}$, Afonso Figueiredo Filho ${ }^{3}$, Andrea Nogueira Dias ${ }^{3}$, Luiz Gastão \\ Bernett $^{4}$ e Marco Aurélio Figura ${ }^{4}$
}

\begin{abstract}
RESUMO - Este trabalho teve como objetivo desenvolver modelos de crescimento e produção em classes de diâmetro para plantios de Eucalyptus grandis submetidos a desbastes. Os dados utilizados na modelagem foram provenientes de plantios de Eucalyptus grandis localizados no Município de Telêmaco Borba, Estado do Paraná, e oriundos de parcelas permanentes, temporárias e de inventário pré-corte, com idades variando de 2,5 a 26,5 anos. A função de densidade de probabilidade Weibull foi utilizada para gerar a distribuição diamétrica. O processo de modelagem de atributos do povoamento foi dividido em etapas, considerando-se os estágios de desenvolvimento do povoamento. Dessa maneira, dois sistemas de prognose foram gerados para predizer os atributos na idade do primeiro desbaste, segundo desbaste ou para o corte final. Os atributos modelados foram aqueles necessários para recuperação dos parâmetros da função Weibull nas idades de intervenção pelo método dos momentos. Para avaliação das prognoses, utilizaram-se o teste de aderência de Kolmogorov-Smirnov e o teste de identidade L\&O proposto por Leite e Oliveira (2002). Os dois sistemas de prognose apresentaram boas estimativas de ajuste e precisão. A metodologia empregada possibilitou simular o crescimento e produção em plantios de Eucalyptus grandis ao longo da rotação, gerando resultados adequados, principalmente, para o corte final, quando são colhidas as melhores árvores com maiores valores agregados.
\end{abstract}

Palavras-chave: Projeção da estrutura diamétrica, Desbastes e Função Weibull.

\section{GROWTH AND YIELD PROGNOSIS IN DIAMETER CLASSES FOR THINNING Eucalyptus grandis STANDS IN BRAZIL SOUTH}

\begin{abstract}
This study aimed to develop growth and yield models by diameter classes for Eucalyptus grandis plantation with thinnings. The data used for modeling are from Eucalyptus grandis plantation located in Telêmaco Borba, Parana state, and came from permanent, temporary plots and pre-harvesting inventory, with ages ranging from 2.5 to 26.5 years. The Weibull probability density function was used to generate the diameter distribution. The stand attributes modeling process was divided into steps, considering the development stages of stands. Thus, two prognosis systems were generated to predict the attributes in the age of first thinning, second thinning or for the final cut. The modeled attributes were those required for recovery the Weibull function parameters at the ages of intervention by the moments method. To evaluate the prognoses the KolmogorovSmirnov adherence test and the L\&O identity test proposed by Leite and Oliveira (2002) were used. Both prognosis systems presented good estimates of adjustment and accuracy. The methodology allowed to simulate the growth and yield in Eucalyptus grandis stands trough the rotation, generating logical results, especially for the final cut where the best trees was harvested with higher added values.
\end{abstract}

Keywords: Diametric structure projection, Thinning and Weibull function.

\footnotetext{
${ }^{1}$ Recebido em 06.10.2011 e aceito para publicação em 28.05.2012.

${ }^{2}$ Curso de Pós-Graduação em Engenharia Florestal da UNICENTRO, Irati (PR). E-mail: <faretslaff@ gmail.com>.

${ }^{3}$ Departamento de Engenharia Florestal do Curso de Engenharia Florestal da UNICENTRO, Irati (PR). E-mail: <afonso.figueiredo@pq.cnpq.br>e<anogueiradias@hotmail.com>.

${ }^{4}$ Klabin SA, Av. Araucária, Telêmaco Borba (PR). E-mail: <gastao@klabin.com.br>e <mafigura@klabin.com.br>.
} 


\section{INTRODUÇÃO}

Os pesquisadores da área florestal têm buscado novas técnicas para aumentar a produtividade das florestas e a qualidade de seus produtos. Algumas das técnicas silviculturais mais utilizadas nos plantios florestais são a poda e o desbaste, e a sua utilização depende da espécie, sítio e objetivos do plantio, salientando que a intensidade e qualidade da aplicação dessas técnicas influenciarão os produtos obtidos.

Os gestores florestais buscam obter relações quantitativas ou modelos que sejam compatíveis, lógicos e forneçam informações consistentes sobre o desenvolvimento futuro dos povoamentos florestais, sejam estes sujeitos ou não a desbastes (SCOLFORO, 1990).

A modelagem do crescimento e da produção florestal busca prognosticar a produção em volume, em área basal, ou em peso em função de uma série de variáveis que são possíveis de serem quantificadas no povoamento florestal (SCOLFORO, 1998).

Em uma empresa florestal, o planejamento da produção é fundamental e, se o objetivo é obter multiprodutos da floresta, o uso de modelos por classe diamétrica possibilita a análise detalhada a respeito da estrutura do povoamento e do porte das árvores via simulação de uma série de regimes de manejo que auxiliam, sobremaneira, na decisão acerca dos momentos adequados de intervenção e na definição da melhor alternativa de rotação para cada unidade de manejo.

As distribuições de diâmetros descrevem a estrutura da floresta (MALTAMO et al., 2000), e a análise da distribuição diamétrica permite estimar o número de árvores por hectare de cada classe de diâmetro, assim como determinar a altura média nas classes de diâmetro, possibilitando, desse modo, a obtenção de informações mais detalhadas sobre a estrutura de produção do povoamento. Esses dados, quando usados em conjunto com uma equação de volume individual, possibilitam estimar a produção por classe de diâmetro e por unidade de área (CLUTTER et al., 1983).

Os modelos de distribuição diamétrica são importantes para situações em que se tem interesse por multiprodutos de madeira. Sua principal característica é o emprego de uma função de densidade de probabilidade $(f d p)$, e a mais comumente utilizada é a função Weibull
(CAMPOS; LEITE, 2009). A projeção da distribuição diamétrica e estimativas de volume de madeira em diferentes classes fornecem uma base quantitativa para a avaliação econômica e o gerenciamento de diferentes opções de manejo (TRINCADO et al., 2003).

Os plantios de eucaliptos no Brasil sempre foram conduzidos sem desbaste, mas nos últimos anos essa prática, aplicada principalmente aos plantios de Pinus, vem sendo utilizada também nos plantios de eucalipto com o objetivo de obter multiprodutos, vislumbrando maiores retornos econômicos. As pesquisas na área de tecnologia da madeira têm comprovado o potencial da madeira de eucalipto para serraria, o que aumentou o interesse do setor moveleiro por árvores de grande porte dessa espécie. A busca do uso múltiplo das florestas e de melhor remuneração para a madeira tem incentivado o estabelecimento de estratégias por parte das empresas para a oferta de madeira serrada de Eucalyptus (SOARES, 2002).

A modelagem de interferências nos povoamentos florestais requer, acima de tudo, a exata definição das operações silviculturais a serem simuladas. Em plantações homogêneas, um parâmetro é frequentemente suficiente para a descrição com precisão alta de um desbaste "por baixo", no qual são removidas somente as menores árvores. Já a modelagem das modificações no povoamento é mais complexa quando o programa silvicultural não é somente restrito a desbastes "por baixo", mas também incluem diferentes tipos de cenários "mistos". Essa situação requer para cada desbaste a descrição do tipo deste, o qual é definido como a remoção seletiva de indivíduos para dada população de árvores (ÁLVAREZ et al., 2002).

São várias as contribuições técnico-científicas sobre estudo de desbastes, mas, segundo Nogueira (2003), as hipóteses elaboradas para o crescimento e produção de florestas equiâneas desbastadas e as principais técnicas desenvolvidas foram, muitas vezes, fundamentadas em dados de povoamentos não desbastados, especialmente de Pinus e, em alguns casos, de eucaliptos. Decisões relacionadas ao regime de manejo de florestas desbastadas necessitam de informações de crescimento e produção que incorporem respostas do desbaste (PIENAAR, 1979).

Como mencionado, algumas plantações de eucaliptos vêm sendo manejadas com desbastes nos últimos anos, e alguns trabalhos de simulação do 
crescimento têm sido conduzidos, entre eles podem ser citados: Dias et al. (2005), Nogueira et al. (2001), Nogueira et al. (2005) e Santana (2008).

Nesse contexto, este trabalho teve como objetivo principal desenvolver modelos de crescimento e produção em classes de diâmetro para plantios de Eucalyptus grandis submetidos a desbastes.

\section{MATERIAL E MÉTODOS}

\subsection{Origem e detalhamento dos dados}

Os dados utilizados neste estudo são provenientes de plantios de Eucalyptus grandis localizados no Município de Telêmaco Borba, Estado do Paraná, disponibilizados pela Klabin S. A.

São oriundos de parcelas permanentes, temporárias e de inventário pré-corte, com área variando de 375 a $900 \mathrm{~m}^{2}$. Em cada parcela foram medidas todas as circunferências a $1,30 \mathrm{~m}$ de altura do solo, as alturas totais das 10 primeiras árvores e das quatro a nove árvores dominantes, de acordo com a área da parcela, segundo o conceito de ASSMANN para altura dominante. As idades dos plantios variaram de 2,5 a 26,5 anos.

\subsection{Processos de simulação}

O processo de modelagem foi dividido em etapas, considerando os estágios de desenvolvimento do povoamento. Em uma etapa preliminar foram utilizadas equações hipsométricas e de sítio geradas por Retslaff (2010) e uma função de afilamento disponibilizada pela empresa Klabin S. A. A função de densidade de probabilidade Weibull foi escolhida para gerar a distribuição diamétrica, como se segue:

$$
f(x)=\left(\frac{c}{b}\right)\left(\frac{x-a}{b}\right)^{c-1} \exp \left[-\left(\frac{x-a}{b}\right)^{c}\right]
$$

em que $f(x)=$ função de densidade de probabilidade Weibull; $a$ = parâmetro de locação; $b=$ parâmetro de escala; $c=$ parâmetro de forma; e $x=$ variável de interesse (diâmetro). Com $a \leq x \leq \infty, a \geq 0, b>0$ e $c>0$.

Para a recuperação dos parâmetros da função Weibull nas idades de intervenção, um método de ajuste da função Weibull foi integrado a um sistema de prognose de atributos da plantação. Dessa forma, foram gerados dois sistemas de prognose, seguindo-se a metodologia utilizada por Santana (2008), em que um primeiro sistema foi utilizado para predição de atributos na idade do primeiro desbaste (D1), e o outro sistema foi empregado para a projeção de atributos na idade de intervenção do segundo desbaste (D2) ou corte final (CF). No sistema de predição, valores atuais são utilizados para estimar os atributos do povoamento, já no sistema de projeção os atributos do povoamento são projetados de uma idade atual para uma idade futura, sendo necessário para o ajuste dos modelos dados de remedição.

Tornou-se, desse modo, possível recuperar a distribuição diamétrica teórica na idade de desbaste ou no corte final. Os atributos modelados foram aqueles necessários para a recuperação dos parâmetros da função Weibull pelo Método dos Momentos nas idades de intervenção (D1, D2 e CF).

Na sequência são apresentadas as etapas realizadas para a simulação do crescimento e da produção por classes de diâmetro:

\section{a) Recuperação dos parâmetros da Função Weibull}

O Método dos Momentos utilizado para estimativa dos parâmetros da função Weibull permite estimar os três parâmetros simultaneamente, com base nas seguintes equações (ARCE, 2004):

Parâmetro “ $a$ ” (localização)

$$
a=\frac{\left[\bar{d}-\left(d g-D_{\min }\right)\right]}{\left[1-\frac{1}{n^{\left(1+\frac{1}{c}\right)}}\right]}
$$

Parâmetro " $b$ " (escala):

$$
b=\left[\frac{\bar{d}-D_{\min }}{\Gamma\left(1+\frac{1}{c}\right)}\right] \cdot\left[1-\frac{1}{n^{\left(1+\frac{1}{c}\right)}}\right]
$$

Parâmetro "c" (forma):

$$
d g^{2}=\bar{d}^{2}+\left(\bar{d}-D_{\min }\right)^{2} \cdot \frac{\left[\Gamma\left(1+\frac{2}{c}\right) /\left[\Gamma\left(1+\frac{1}{c}\right)^{2}\right]\right]-1}{\left[1-\left(\frac{1}{n}\right)^{\left(1+\frac{1}{c}\right)}\right]^{2}}
$$

Revista Árvore, Viçosa-MG, v.36, n.4, p.719-732, 2012 
em que $\bar{d}=$ diâmetro médio aritmético $(\mathrm{cm}) ; d g=$ diâmetro médio quadrático $(\mathrm{cm}) ; D_{\min }=$ diâmetro mínimo $(\mathrm{cm})$; $n=$ número de diâmetros; $\Gamma=$ função gama; $a=$ parâmetro de locação; $b=$ parâmetro de escala; e $c=$ parâmetro de forma.

Nota-se que a expressão (4) é função apenas do parâmetro " $c$ " e pode ser resolvida por alguma técnica de iteração, desde que sejam conhecidos o $d g$, a média aritmética dos diâmetros $(\bar{d})$, o diâmetro mínimo $\left(D_{\text {min }}\right)$ e o número de diâmetros $(n)$. Assim, estimado o parâmetro " $c$ ", podem-se estimar os parâmetros " $a$ " e " $b$ ", resolvendo as expressões (2) e (3), respectivamente (ARCE, 2004).

Dessa maneira, foram utilizados modelos para estimar os atributos do povoamento $D_{\min }, \bar{d}$ e $d g$ nas diferentes idades. Com a obtenção desses atributos, foi utilizado o método iterativo de Newton para estimativa do parâmetro de forma " $c$ "; então, os parâmetros " $a$ " e " $b$ " puderam ser obtidos, resolvendo-se as equações (2) e (3).

\section{b) Sistema de equações para predição de atributos na idade do primeiro desbaste (D1)}

Nesta etapa foram testados modelos para obter um sistema de equações para estimar os atributos/características dos plantios na idade do primeiro desbaste (D1).

Para estimar o número de árvores nos povoamentos antes do primeiro desbaste foram testados sete modelos de sobrevivência. Os modelos de sobrevivência foram ajustados com dados de povoamentos sem desbastes, tendo sido utilizadas informações do número de árvores sobreviventes oriundas de 46 parcelas permanentes sem desbaste.

Além dos modelos que estimam a sobrevivência com dados de parcelas permanentes, foram testados modelos que utilizam o número de árvores sobreviventes em função do número de árvores plantadas, considerando-se o número de árvores plantadas como o número de árvores da parcela menos o número de falhas de plantio. O número de árvores sobreviventes foi considerado como o número de árvores da parcela menos as árvores marcadas como mortas. No ajuste desses modelos foram utilizados dados de 227 parcelas.

Os modelos testados para estimar sobrevivência e os atributos/características dos plantios na idade do primeiro desbaste estão apresentados na Tabela 1. Os modelos identificados nas tabelas com um "traço"
(-) no campo Autor/Fonte foram construídos buscando melhoria nos ajustes. Isso foi feito com base na correlação linear entre as variáveis dependentes e outras variáveis do povoamento, como: idade $(I)$, altura dominante $\left(h_{d o m}\right)$, diâmetro médio quadrático $(d g)$, índice de sítio $(S)$, diâmetro médio aritmético $(\bar{d})$ e número de árvores por hectare $(N)$. A permanência ou não da variável no modelo se deu em função do $p$-valor obtido para o coeficiente ligado a essa variável $(p<0,05)$.

O diâmetro médio quadrático $(d g)$ e o diâmetro médio aritmético $(\bar{d})$ foram obtidos pelas fórmulas:

$$
\begin{gathered}
d g=\sqrt{\frac{G}{\left(\frac{\pi}{40000}\right) \cdot N}} \\
\bar{d}=\left\{d g^{2}-\exp \left[\left(\ln \left(S^{2} d\right)\right]\right\}^{\left(\frac{1}{2}\right)}\right.
\end{gathered}
$$

\section{c) Desbastes}

Para obter a quantidade de árvores a serem removidas em cada classe de diâmetro, adotou-se o seguinte modelo utilizado por Scolforo (1990):

$$
P_{i}=\exp \left[b_{1}\left(\frac{d_{i}^{2}}{d g^{2}}\right)^{b_{2}}\right]
$$

em que $P_{i}=$ proporção de árvores/ha removidas na classe de diâmetro $i ; d_{i}=$ centro da classe de diâmetro $i ; d g$ $=$ diâmetro médio quadrático $(\mathrm{cm}) ; \mathrm{e} b_{1}, b_{2}=$ coeficientes estimados a partir dos dados.

Os ajustes foram realizados separadamente para o primeiro e o segundo desbaste. Os dados utilizados para o ajuste da equação de desbaste foram obtidos de parcelas de inventário pré-corte (IPC). Em cada parcela foi feito agrupamento dos dados em classes de diâmetro com amplitude de $2 \mathrm{~cm}$, imediatamente antes de realizar o desbaste.

O número de árvores por hectare antes do D1 variou de 832 a 1.586 árvores/ha, com idades entre 5,3 e 7,4 anos. O número de árvores remanescente variou de 400 a 944 árvores/ha depois do D1. Já os dados para o ajuste do D2 variaram de 347 a 896 árvores/ha antes do D2, com um número de árvores remanescente por hectare de 208 a 432. As idades em que foi realizado o D2 variaram de 7,9 a 12,1 anos. 
Prognose do crescimento e da produção em classes de...

Tabela 1 - Modelos testados para estimar sobrevivência $\left(N_{2}\right)$, área basal $(G)$, diâmetro mínimo $\left(D_{\text {min }}\right)$, variância dos diâmetros $\left(S^{2} d\right)$ e diâmetro máximo $\left(D_{\max }\right)$.

Table 1 - Tested models to estimate survival $\left(N_{2}\right)$, basal area $(G)$, minimum diameter $\left(D_{\min }\right)$, variance of the diameters $\left(S^{2} d\right)$ and maximum diameter $\left(D_{\text {max }}\right)$.

\begin{tabular}{cl}
\hline Autor/Fonte & Modelo \\
\hline Sobrevivência $\left(N_{2}\right)$ & Eq. \\
Pienaar e Shiver/Scolforo (1998) & $\ln \left(N_{2}\right)=\ln \left(N_{1}\right)-\beta_{1}\left(I_{2} \beta_{2}-I_{1}^{\beta_{2}}\right)$ \\
Lenhart/Scolforo (1998) & $N_{2}=N_{1} \exp \left[\beta_{1}\left(I_{2}-I_{1}\right)+\beta_{2}\left(\ln \left(\frac{I_{2}}{I_{1}}\right)\right]\right]$ \\
Clutter/Scolforo (1998) & $\left.N_{2}=N_{1}\left(\frac{I_{2}}{I_{1}}\right) \cdot \exp \left(\beta_{0}+\beta_{2} S\right)\left(I_{2}-I_{1}\right)\right]$ \\
Acerbi Jr. et al. (2002) & $N_{2}=N_{1}\left\{\exp \left[I_{2}\left(\beta_{1}+\beta_{2} \ln \left(N_{1}\right)+\beta_{3} S\right)\right]\right\}$ \\
Feduccia et al. (1979) & $N_{s}=N_{p} /\left[\exp \left(I\left(\beta_{1} \ln \left(N_{p}\right)+\beta_{2} h_{d o m}+\beta_{3}\left(h_{d o m}{ }^{1 / 2}\right)\right)\right]\right.$ \\
Burkhart e Sprinz (1984) & $N_{s}=\left\{N_{p} \cdot\left[\exp \left(I\left(\beta_{0}-\beta_{1} \ln \left(N_{p}\right)\right)\right]\right\}\right.$ \\
Acerbi Jr. et al. (2002) Modificado & $N_{s}=N_{p}\left\{\exp \left[I_{2}\left(\beta_{1}+b_{2} \ln \left(N_{1}\right)+\beta_{3} S\right)\right]\right\}$ \\
\hline & Modelos para estimar área basal $(\boldsymbol{G})$
\end{tabular}

Scolforo (1998)

$\ln (G)=\beta_{0}+\beta_{1}\left(\frac{1}{I}\right)+\beta_{2} S+\beta_{3} \ln \left(\frac{1}{N}\right)$

Santana (2008)

$G=\beta_{0}+\beta_{1} \ln (I)+\beta_{2} \ln (N)$

Scolforo(1990)

$\ln (G)=\beta_{0}+\frac{\beta_{1}}{I}+\beta_{2} \ln (N)+\beta_{3} \ln (S)+\beta_{4} \ln \left(\frac{N}{I}\right)+\beta_{5} \ln (S \cdot I)$

Scolforo (1990)

$\ln (G)=\beta_{0}+\beta_{1}\left(\frac{1}{I}\right)+\beta_{2} S+\beta_{3}\left(\frac{1}{N}\right)$

Scolforo (1990)

$\ln (G)=\beta_{0}+\beta_{1} \ln (N)+\beta_{2}(I)+\beta_{3}\left(\frac{1}{I}\right)+\beta_{4}\left(\frac{1}{S}\right)$

Scolforo (1998)

$G=\beta_{0}+\beta_{1} N+\beta_{2} h_{\text {dom }}$

\section{Modelos para estimar Diâmetro mínimo $\left(D_{\mathrm{min}}\right)$}

Santana (2008)

$D_{\min }=\beta_{0}+\beta_{1} \bar{d}+\beta_{2} h_{d o m}+\beta_{3} \sqrt{S^{2} d}+\beta_{4} \ln (G)+\beta_{5} d g$

Scolforo/Scolforo (1998)

$D_{\min }=\beta_{0}+\beta_{1} I+\beta_{2}\left(\frac{h_{d o m}}{I}\right)+\beta_{3} N+\beta_{4} d g$

Cao et al./Scolforo (1998)

$D_{\text {min }}=\beta_{0}+\frac{\beta_{1}}{I}+\beta_{2} \ln \left(h_{d o m}\right)+\beta_{3} \ln (G)+\beta_{4} \ln (N)$

- - - - - - - - - - - - - - - - - - - - - - - - - -

Continued... 
Tabela 1 - Cont.

Table 1 - Cont

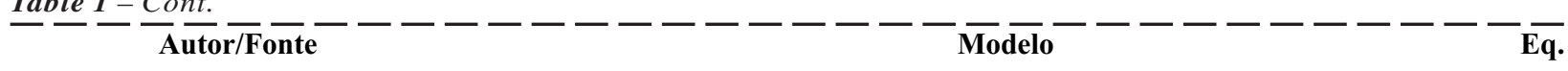

Sobrevivência $\left(\mathrm{N}_{2}\right)$

Lenhart/Scolforo (1998)

Burkhart e Daniels/Scolforo (1998)

Burkhart e Sprinz/Scolforo (1998)

$$
\ln \left(D_{\min }\right)=\beta_{0}+\beta_{1} h_{d o m}+\beta_{2} N
$$$$
D_{\text {min }}=\beta_{0}+\beta_{1} h_{d o m}+\beta_{2}\left(\frac{h_{d o m}}{N}\right)
$$$$
D_{\text {min }}=\beta_{0}+\beta_{1} h_{d o m}+\beta_{2}(I \cdot N)+\beta_{3}\left(\frac{h_{d o m}}{N}\right)+\beta_{4}(D g \cdot I)
$$$$
\ln \left(D_{\min }\right)=\beta_{0}+\beta_{1} I+\beta_{2}\left(\frac{h_{\text {dom }}}{I}\right)+\beta_{3} N
$$

\section{Modelos para estimar a variância dos diâmetros $\left(S^{2} d\right)$}

Burk e Burkhart

Modificado/Scolforo (1998)

$$
\ln \left(d g^{2}-\bar{d}^{2}\right)=\ln \left(S^{2} d\right)=\beta_{0}+\beta_{1} \ln \left(h_{\text {dom }}\right)+\frac{\beta_{2}}{h_{\text {dom }}}+\beta_{3} \ln (G)+\frac{\beta_{4}}{G}
$$

Knoebell e Burkhart/Scolforo (1998)

$$
\ln \left(d g^{2}-\bar{d}^{2}\right)=\beta_{0}+\beta_{1} \ln (G)+\beta_{2} \ln \left(h_{d o m}\right)+\beta_{3}(I \cdot N)
$$

Scolforo 1/Scolforo (1990)

$$
\ln \left(d g^{2}-\bar{d}^{2}\right)=\ln \left(S^{2} d\right)=\beta_{0}+\beta_{1} \ln \left(h_{d o m}\right)+\beta_{2}\left(\frac{1}{h_{d o m}}\right)+\beta_{3} \ln (G)
$$

Scolforo 2/Santana (2008)

$$
\ln \left(d g^{2}-\bar{d}^{2}\right)=\ln \left(S^{2} d\right)=\beta_{0}+\beta_{1} \ln \left(h_{d o m}\right)+\beta_{2} \ln \left(\frac{G}{N}\right)+\beta_{3}\left(\frac{h_{d o m}}{I}\right)
$$

\section{Modelos para estimar diâmetro máximo ( $\left.D_{\text {máx }}\right)$}

Scolforo (1998)

Wendling (2007)

Santana (2008)

Modificado

$$
D_{\text {máx }}=\beta_{1} I+\beta_{2} S+\beta_{3} d g
$$$$
D_{\text {máx }}=\beta_{1} I+\beta_{2} h_{\text {dom }}+\beta_{3} N+\beta_{4} d g
$$

Em que $N_{2}=$ número de árvores/ha na idade $2 ; N_{1}=$ número de árvores/ha na idade $1 ; I=$ idade 1 (anos); $\mathrm{I}_{2}=$ idade 2 (anos); $N=$ número de árvores da medição na idade $I ; N=$ número de árvores/ha plantadas menos falhas de plantio; exp = exponencial; $G=$ área basal $\left(\mathrm{m}^{2} / \mathrm{ha}\right) ; I=$ idade $\left(\right.$ anos) $; S=$ índice de ${ }^{p}$ sítio $(\mathrm{m}) ; N=$ número de árvores por hectare; $h_{d o m}=$ altura média das árvores dominantes $(\mathrm{m}) ; D_{\min }=$ diâmetro mínimo $(\mathrm{cm}) ; I=$ idade $(\operatorname{anos}) ; S^{2} d=$ variância dos diâmetros $\left(\mathrm{cm}^{2}\right) ; d g=$ diâmetro médio quadrático $(\mathrm{cm}) ; \bar{d}$ = diâmetro médio $(\mathrm{cm}) ; D_{\max }=$ diâmetro máximo $(\mathrm{cm}) ; \beta_{i s}=$ coeficientes a serem estimados; e Eq.= número da equação.

\section{d) Sistema de equações para projeção de atributos na} idade do segundo desbaste (D2) e no corte final

Para projetar os parâmetros da função Weibull depois dos desbastes utilizando o Método dos
Momentos, foi necessário projetar os atributos requeridos por esse método, ou seja, foram projetados para a próxima idade de intervenção o diâmetro mínimo $\left(D_{\text {min }}\right)$, o diâmetro médio quadrático $(d g)$ e o diâmetro

Revista Árvore, Viçosa-MG, v.36, n.4, p.719-732, 2012 
médio aritmético $(\bar{d})$ remanescentes após o desbaste. Para isso, dados das parcelas permanentes foram utilizados para ajustar modelos que projetam esses atributos/características para uma próxima intervenção (desbaste ou corte final). Os modelos testados são apresentados na Tabela 2 .

A forma matemática do modelo de sobrevivência $\left(\mathrm{N}_{2}\right)$ proposto por Pienaar e Shiver (1981) foi utilizada por Nogueira (2003) para estimar o diâmetro mínimo $\left(D_{\text {min } 2}\right)$. Neste trabalho, além de testar essa alternativa para projetar $D_{\min 2}$, ela também foi testada para projetar a variância dos diâmetros $\left(S^{2} d_{2}\right)$ e o diâmetro máximo $\left(D_{\max 2}\right)$.

\subsection{Análise e seleção dos modelos}

Para análise e seleção dos modelos ajustados em todas as etapas do processo de simulação, foram utilizadas as seguintes estatísticas: Coeficiente de determinação ajustado $\left(R_{a d j}^{2}\right)$, Erro-padrão de estimativa $\left(S_{y x} \%\right)$ para a variável de interesse e a Análise gráfica da distribuição de resíduos (Res\%).

\subsection{Avaliação da projeção da produção em classes de diâmetro obtidas antes e depois de cada intervenção}

O teste de aderência de Kolmogorov-Smirnov (K-S) foi utilizado para avaliar a distribuição estimada. O teste K-S compara a frequência acumulada estimada com a frequência acumulada observada. O valor $D$ de K-S é o ponto de máxima divergência (máxima diferença) entre a distribuição estimada e a distribuição observada (SCOLFORO, 1998; BARRA et al., 2004). Utilizou-se o nível de significância de $1 \%$.

Para a comparação dos volumes prognosticados com os volumes observados por classe diamétrica, utilizou-se o teste de identidade L\&O proposto por Leite e Oliveira (2002), cujo procedimento resulta da combinação do teste de F(H0) Graybill, do teste t para erro médio (t) e da análise de correlação linear $\left(r_{y j y l}\right)$ entre os valores observados $\left(Y_{j}\right)$ e os valores estimados $\left(Y_{l}\right)$. No teste, consideraram-se o valor observado como variável dependente e o valor estimado como variável independente. Em todos os casos, o nível de significância adotado foi $1 \%$.

\section{RESULTADOS}

\subsection{Sistemas de equações para predição e projeção de atributos}

Na Tabela 3 estão reunidos os melhores modelos selecionados pela análise do coeficiente de determinação ajustado, erro-padrão de estimativa e distribuição gráfica dos resíduos. Esses modelos foram empregados para estimar cada variável antes de cada intervenção, e a equação (36) foi utilizada para simular os desbastes.

\subsection{Estimativas dos atributos para recuperação dos parâmetros da função Weibull nas idades de desbastes e corte final}

As estimativas dos atributos/características do povoamento foram obtidas imediatamente antes de cada desbaste (D1 e D2) e corte final (CF) para cinco índices de sítio, obtidos com o modelo ajustado em Retslaff (2010).

Com os modelos selecionados no sistema de equações para predição de atributos (Tabela 3), foram estimados os atributos do povoamento para cada índice de sítio e recuperados os parâmetros da função Weibull na idade do primeiro desbaste (Tabela 4). A partir dos parâmetros obtidos para cada um dos índices de sítios, gerou-se a distribuição diamétrica teórica de diâmetros, tendo sido comparadas com os dados médios por sítio, quando havia dados disponíveis para isso.

As estimativas de $N / h a, G$ e volume por classes diamétricas obtidas imediatamente antes do primeiro desbaste foram estatisticamente iguais para os índices de sítio 38 e 43 m e estatisticamente diferentes para o índice de sítio de $48 \mathrm{~m}$ por meio do teste L\&O (para nível de significância de 1\%). Nos demais sítios não havia dados disponíveis para comparação.

As distribuições estimadas com os parâmetros da função Weibull recuperados para os três índices de sítio $(S=38 \mathrm{~m}, S=43 \mathrm{~m}$ e $S=48 \mathrm{~m})$, imediatamente antes do primeiro desbaste, foram aceitas pelo teste $\mathrm{K}-\mathrm{S}$ (resultado do teste não significativo a $1 \%$ de probabilidade).

Após a obtenção da distribuição imediatamente antes do primeiro desbaste, foi simulado o primeiro desbaste (em torno dos seis anos). As comparações

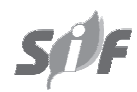

Revista Árvore, Viçosa-MG, v.36, n.4, p.719-732, 2012 
Tabela 2 - Modelos testados para projetar a área basal $\left(G_{2}\right)$, a variância dos diâmetros $\left(S^{2} d_{2}\right)$, o diâmetro mínimo $\left(D_{\text {minz }}\right)$ e o diâmetro máximo $\left(D_{\max 2}\right)$.

Table 2 - Tested models to project the basal area $\left(G_{2}\right)$, the variance of the diameters $\left(S^{2} d_{2}\right)$, the minimum diameter $\left(D_{\text {min } 2}\right)$, and maximum diameter $\left(D_{\max 2}\right)$.

\begin{tabular}{|c|c|c|}
\hline Autor/Fonte & Modelo & Eq. \\
\hline \multicolumn{3}{|c|}{ Área basal $\left(G_{2}\right)$} \\
\hline $\begin{array}{l}\text { Scolforo 1/Santana } \\
\qquad(2008)\end{array}$ & $G_{2}=\beta_{1} G_{1}\left(\frac{I_{1}}{I_{2}}\right)+\beta_{2}\left(1-\frac{I_{1}}{I_{2}}\right) \ln \left(N_{2}\right)+\beta_{3}\left(1-\frac{I_{1}}{I_{2}}\right) \ln \left(h_{\text {dom } 2}\right)+\beta_{4}\left(1-\frac{I_{1}}{I_{2}}\right) \ln \left(I_{2}\right)$ & $(37)$ \\
\hline $\begin{array}{l}\text { Scolforo 2/Santana } \\
\quad(2008)\end{array}$ & $G_{2}=\beta_{1} G_{1}\left(\frac{I_{1}}{I_{2}}\right)+\beta_{2}\left(1-\frac{I_{1}}{I_{2}}\right) \ln \left(N_{2}\right)+\beta_{3}\left(1-\frac{I_{1}}{I_{2}}\right) \ln \left(h_{\text {dom } 2}\right)+\beta_{4}\left(1-\frac{I_{1}}{I_{2}}\right) I_{2}^{2}$ & $(38)$ \\
\hline Clutter (1963) & $\ln \left(G_{2}\right)=\ln \left(G_{1}\right)\left(\frac{I_{1}}{I_{2}}\right)+\beta_{1}\left(1-\frac{I_{1}}{I_{2}}\right)+\beta_{2}\left(1-\frac{I_{1}}{I_{2}}\right) S$ & $(39)$ \\
\hline \multicolumn{3}{|c|}{ Variância dos diâmetros $\left(S^{2} d_{2}\right)$} \\
\hline Santana (2008) & $S^{2} d_{2}=\beta_{1} S^{2} d_{1}+\beta_{2} G_{1}+\beta_{3} G_{2}$ & $(40)$ \\
\hline $\begin{array}{l}\text { Nogueira }(2003) \\
\text { modificado }\end{array}$ & $S^{2} d_{2}=S^{2} d_{1}$ & $(41)$ \\
\hline- & $S^{2} d_{2}=\beta_{0}+\beta_{1}\left(1-\frac{I_{1}}{I}\right)+\beta_{2} D_{\min _{2}}+\beta_{3} S^{2} d_{1}+D_{\text {máx } 2}$ & $(42)$ \\
\hline \multicolumn{3}{|c|}{ Diâmetro mínimo $\left(D_{\min 2}\right)$} \\
\hline Nogueira (2003) & $D_{\min 2}=D_{\min 1} e^{\left(-\beta_{1} \cdot\left(I_{2} \beta_{2}-I_{1} \beta_{2}\right)\right)}$ & $(43)$ \\
\hline $\begin{array}{c}\text { Scolforo } \\
\text { (SANTANA, 2008) }\end{array}$ & $D_{\min 2}=\beta_{0}+\beta_{1} \bar{d}_{2}+\beta_{2} h_{d o m 2}+\beta_{3} S d_{2}+\beta_{4} \ln \left(G_{2}\right)+\beta_{5}\left(I_{2}\right)$ & $(44)$ \\
\hline $\begin{array}{c}\text { Abreu } \\
\text { (SANTANA, 2008) }\end{array}$ & $D_{\min 2}=\beta_{0}+\beta_{1} \bar{d}_{2}+\beta_{2} h_{d o m 2}+\beta_{3} S d_{2}+\beta_{4} \ln \left(G_{2}\right)+\beta_{5}\left(d g_{2}\right)$ & $(45)$ \\
\hline- & $D_{\min 2}=\beta_{1} D_{\min 1}+\beta_{2} \frac{I_{1}}{I_{2}}$ & $(46)$ \\
\hline \multicolumn{3}{|c|}{ Diâmetro máximo $\left(D_{\operatorname{máx} 2}\right)$} \\
\hline $\begin{array}{l}\text { Nogueira (2003) } \\
\text { Modificado }\end{array}$ & $D_{\text {máx } 2}=D_{\text {máx } 1} e^{\left(-\beta_{1} \cdot\left(I_{2}^{\beta_{2}}-I_{1}^{\beta_{2}}\right)\right)}$ & $(47)$ \\
\hline $\begin{array}{c}\text { Santana } \\
(2008)\end{array}$ & $D_{\text {máx } 2}=D_{\text {máx } 1}\left(\frac{I_{1}}{I_{2}}\right)+\beta_{1}\left(1-\frac{I_{1}}{I_{2}}\right) d g_{2}$ & $(48)$ \\
\hline $\begin{array}{l}\text { Santana } \\
(2008)\end{array}$ & $D_{\max 2}=\beta_{0}+\beta_{1}\left(\frac{I_{1}}{I_{2}}\right) \ln \left(h_{\text {dom } 2}\right)+\beta_{2}\left(\frac{I_{1}}{I_{2}}\right) \ln \left(I_{2}\right)+\beta_{3}\left(\frac{I_{1}}{I_{2}}\right) \ln \left(N_{2}\right)+\beta_{4}\left(\frac{I_{1}}{I_{2}}\right) \ln \left(G_{2}\right)$ & $(49)$ \\
\hline- & $D_{\text {máx } 2}=\beta_{0}+\beta_{1} D_{\text {máx } 1}+\beta_{2}\left(1-\frac{I_{1}}{I_{2}}\right)$ & $(50)$ \\
\hline
\end{tabular}

Em que $G_{1}=$ área basal na idade $1\left(\mathrm{~m}^{2} / \mathrm{ha}\right) ; G_{2}=$ área basal na idade $2\left(\mathrm{~m}^{2} / \mathrm{ha}\right) ; N_{2}=$ número de árvores na idade $2(\mathrm{~N} / \mathrm{ha}) ; S=$ índice de $\operatorname{sítio}(\mathrm{m}) ; I_{1}=$ idade inicial da plantação (anos); $I_{2}=$ idade futura da plantação (anos); $h_{d o m} 2=$ altura média das árvores dominantes na idade $2(\mathrm{~m}) ; S^{2} d_{2}=$ variância dos diâmetros na idade $2\left(\mathrm{~cm}^{2}\right) ; S^{2} d=$ variância dos diâmetros na idade $\left.1(\mathrm{~cm})^{2}\right) ; \bar{d}=$ média aritmética dos diâmetros na idade $2(\mathrm{~cm}) ; d g=$ diâmetro médio quadrático na idade $2 ; D$ = diâmetro mínimo na idade $2(\mathrm{~cm}) ; D$, diâmetro máximo na idade $2(\mathrm{~cm}) ; D_{\text {minl }}=$ diâmetro mínimo na idade $1(\mathrm{~cm}) ; S d_{2}=$ desvio-padrão dos diâmetros na idade $2(\mathrm{~cm}) ; D_{m a ́ x l}=$ diâmetro máximo na idade $1(\mathrm{~cm}) ; \beta_{i s}=$ coeficientes a serem estimados; e Eq. = número da equação.

Revista Árvore, Viçosa-MG, v.36, n.4, p.719-732, 2012 
Tabela 3 - Modelos selecionados para estimar os atributos/características do povoamento imediatamente antes de cada intervenção e modelo para simular os desbastes com os respectivos coeficientes de determinação ( $R^{2}$ adj) e erro-padrão de estimativa $($ Syx $\%)$.

Table 3 - Selected models for estimating attributes/characteristics of the stand immediately before each intervention and model to simulate the thinning with respective coefficients of determination $\left(R_{a d j}^{2}\right)$ and standard error of estimate $\left(S_{y x}\right)$.

\begin{tabular}{|c|c|c|c|c|c|c|}
\hline Autor/Fonte & Modelo & Coeficientes & $\mathbf{R}^{2} \mathbf{a d j}$ & Syx (\%) & $\mathbf{n}$ & Eq. \\
\hline \multicolumn{7}{|c|}{ Sistema de equações para predição de atributos } \\
\hline $\begin{array}{l}\text { Feduccia } \\
\text { et al. (1979) }\end{array}$ & $N_{s}=N_{p} /\left[\exp \left(I\left(\beta_{1} \ln \left(N_{p}\right)+\beta_{2} h_{d o m}+\beta_{3}\left(h_{d o m}^{1 / 2}\right)\right)\right]\right.$ & \multirow{4}{*}{$\begin{array}{l}\beta_{1}=0,00223 \\
\beta_{2}=0,00030 \\
\beta_{3}=-0,00392 \\
\beta_{0}=-1,85116 \\
\beta_{1}=-3,05139 \\
\beta_{2}=0,46553 \\
\beta_{3}=0,59691 \\
\beta_{0}=2,99557 \\
\beta_{1}=3,59640 \\
\beta_{2}=0,05769 \\
\beta_{3}=-3,32997 \\
\beta_{4}=-2,69131 \\
\beta_{0}=-7,44687 \\
\beta_{1}=2,41360 \\
\beta_{2}=22,20361 \\
\beta_{3}=0,53611\end{array}$} & 0,9746 & 2,9 & 227 & (9) \\
\hline $\begin{array}{c}\text { Scolforo } \\
(1990) \\
\text { Modificado }\end{array}$ & $\ln (G)=\beta_{0}+\frac{\beta_{1}}{I}+\beta_{2} \ln \left(\frac{N}{I}\right)+\beta_{3} \ln (S I)$ & & 0,8779 & 13,5 & 505 & $(14)$ \\
\hline $\begin{array}{l}\text { Santana } \\
(2008) \\
\text { Modificado }\end{array}$ & $D_{\min }=\beta_{0}+\beta_{1} \bar{d}+\beta_{2} h_{d o m}+\beta_{3} \ln (G)+\beta_{4} d g$ & & 0,8650 & 18,5 & 505 & $(18)$ \\
\hline $\begin{array}{l}\text { Scolforo 1/ } \\
\text { Scolforo } \\
(1990)\end{array}$ & $\ln \left(S^{2} d\right)=\beta_{0}+\beta_{1} \ln \left(h_{d o m}\right)+\beta_{2}\left(\frac{1}{h_{d o m}}\right)+\beta_{3} \ln (G)$ & & 0,7932 & 35,4 & 505 & $(27)$ \\
\hline $\begin{array}{l}\text { Santana } \\
(2008) \\
\text { Modificado }\end{array}$ & $D_{\text {máx }}=\beta_{0}+\beta_{1} \bar{d}+\beta_{2} h_{d o m}+\beta_{3} \ln (G)+\beta_{4} d g$ & $\begin{array}{l}\beta_{0}=-2,74791 \\
\beta_{1}=-5,72659 \\
\beta_{2}=0,22661 \\
\beta_{3}=1,37156 \\
\beta_{4}=6,56224\end{array}$ & 0,9442 & 7,2 & 505 & $(31)$ \\
\hline \multirow{5}{*}{$\begin{array}{l}\text { Scolforo } \\
(1990)\end{array}$} & \multicolumn{2}{|l|}{ Desbastes } & & & & \\
\hline & \multirow{4}{*}{$P_{i}=\exp \left(b_{1}\left(\frac{d_{i}^{2}}{d g^{2}}\right)\right.$} & \multicolumn{5}{|c|}{ Primeiro desbaste } \\
\hline & & $\begin{array}{l}\mathrm{b}_{1}=-0,74563 \\
\mathrm{~b}_{2}=2,06196\end{array}$ & 0,8752 & 33,4 & 97 & $(36)$ \\
\hline & & \multicolumn{5}{|c|}{ Segundodesbaste } \\
\hline & & $\begin{array}{l}\mathrm{b}_{1}=-0,71472 \\
\mathrm{~b}_{2}=4,18556\end{array}$ & 0,8750 & 37,2 & 37 & $(36)$ \\
\hline \multicolumn{7}{|c|}{ Sistema de equações para projeção de atributos } \\
\hline $\begin{array}{l}\text { Clutter } \\
(1963)\end{array}$ & $\ln \left(G_{2}\right)=\ln \left(G_{1}\right)\left(\frac{I_{1}}{I_{2}}\right)+\beta_{1}\left(1-\frac{I_{1}}{I_{2}}\right)+\beta_{2}\left(1-\frac{I_{1}}{I_{2}}\right) S$ & $\begin{array}{l}\beta_{1}=3,03978 \\
\beta_{2}=0,02237\end{array}$ & 0,9656 & 5,6 & 127 & $(39)$ \\
\hline- & $S^{2} d_{2}=\beta_{0}+\beta_{1}\left(1-\frac{I_{1}}{I_{2}}\right)+\beta_{2} D_{\min _{2}}+\beta_{3} S^{2} d_{1}+\beta_{4} D_{\text {máx }}$ & $\begin{array}{l}\beta_{0}=-13,31483 \\
\beta_{1}=38,24657 \\
\beta_{2}=-0,41523 \\
\beta_{3}=0,97919 \\
\beta_{4}=0,54291\end{array}$ & 0,9506 & 14,3 & 127 & (42) \\
\hline- & $D_{\min 2}=\beta_{1} D_{\min 1}+\beta_{2} \frac{I_{1}}{I_{2}}+\beta_{3} d g_{2}$ & $\begin{array}{l}\beta_{1}=0,87141 \\
\beta_{2}=-3,35286 \\
\beta_{3}=0,21084\end{array}$ & 0,9886 & 6,7 & 127 & (46) \\
\hline- & $D_{\max 2}=\beta_{0}+\beta_{1} D_{\max 1}+\beta_{2}\left(1-\frac{I_{1}}{I_{2}}\right)$ & $\begin{array}{l}\beta_{0}=-2,58350 \\
\beta_{1}=1,06904 \\
\beta_{2}=21,01017\end{array}$ & 0,9845 & 3,6 & 127 & $(50)$ \\
\hline
\end{tabular}

Em que $G=$ área basal $\left(\mathrm{m}^{2} / \mathrm{ha}\right) ; I=$ idade $(\operatorname{anos}) ; S=$ índice de sítio $(\mathrm{m}) ; N=$ número de árvores por hectare; $h_{d o m}=$ altura média das árvores dominantes $(\mathrm{m}) ; D_{\min }=$ diâmetro mínimo $(\mathrm{cm}) ; I=$ idade $(\operatorname{anos}) ; S^{2} d=$ variância dos diâmetros $\left(\mathrm{cm}^{2}\right) ; d g=$ diâmetro médio quadrático $(\mathrm{cm})$; $\bar{d}=$ diâmetro médio $(\mathrm{cm}) ; D=$ diâmetro máximo $(\mathrm{cm}) ; P_{i}=$ proporção de árvores $/$ ha removidas na classe de diâmetro $i ; d=$ centro da classe de diâmetro $i ; G_{1}=$ área basal na idade $1\left(\mathrm{~m}^{2} / \mathrm{ha}\right) ; G_{2}=$ área basal na idade $2\left(\mathrm{~m}^{2} / \mathrm{ha}\right) ; N_{2}=$ número de árvores na idade 2 (N/ha); $S=$ índice de sítio $(\mathrm{m}) ; I_{1}=$ idade inicial da plantação (anos); $I_{2}=$ idade futura da plantação (anos); $h_{\text {dom } 2}^{2}=$ altura média das árvores dominantes na idade $2(\mathrm{~m}) ; S^{2} d_{2}=$ variância dos diâmetros na idade $2\left(\mathrm{~cm}^{2}\right) ; S^{2} d_{1}=$ variância dos diâmetros na idade $1\left(\mathrm{~cm}^{2}\right) ; \bar{d}=$ média aritmética dos diâmetros na idade $2(\mathrm{~cm}) ; d g=$ diâmetro médio quadrático na idade $2 ; D=$ diâmetro mínimo na idade $2(\mathrm{~cm}) ; D$ diâmetro máximo na idade $2(\mathrm{~cm}) ; D_{\min 1}=$ diâmetro mínimo na idade $1(\mathrm{~cm}) ; S d_{2}=$ desvio-padrão dos diâmetros na idade $2(\mathrm{~cm}) ; D_{m a x x l}=$ diâmetro máximo na idade $1(\mathrm{~cm}) ; \beta_{i s}^{\min 1}=$ coeficientes a serem estimados; Eq.= número da equação. 
Tabela 4 - Estimativas dos atributos/características e parâmetros da função Weibull antes de cada intervenção. Table 4-Attributes/characteristics estimated and the Weibull function parameters before each intervention.

\begin{tabular}{cccccccccc}
\hline$S$ & N/ha & Dmin & $\bar{d}$ & $D g$ & $D_{\max }$ & $I$ & $a$ & $b$ & $c$ \\
\hline 33 & 1339,1 & 6,11 & 15,89 & 16,42 & 23,66 & 6,0 & 5,57722 & 11,02535 & 2,53535 \\
38 & 1153,3 & 7,25 & 17,22 & 17,83 & 26,07 & 6,3 & 6,63860 & 11,25838 & 2,29330 \\
43 & 1139,0 & 7,40 & 17,82 & 18,56 & 28,24 & 6,3 & 6,66125 & 11,76262 & 2,11234 \\
48 & 1225,3 & 6,76 & 17,64 & 18,52 & 29,75 & 6,0 & 5,87256 & 12,27718 & 2,01284 \\
53 & 1226,4 & 6,62 & 18,01 & 19,07 & 32,03 & 6,0 & 5,56430 & 12,83509 & 1,89069 \\
\hline \multicolumn{7}{c}{ Antes do segundo desbaste (D2) } \\
\hline 33 & 669,1 & 11,28 & 19,49 & 19,94 & 27,96 & 8,0 & 10,82865 & 9,27704 & 2,05085 \\
38 & 585,9 & 11,55 & 21,17 & 21,70 & 29,95 & 8,1 & 11,02265 & 10,85878 & 2,11767 \\
43 & 564,3 & 11,81 & 22,28 & 22,90 & 32,27 & 8,1 & 11,18377 & 11,82053 & 2,07023 \\
48 & 579,5 & 12,11 & 23,04 & 23,73 & 34,67 & 8,1 & 11,41542 & 12,33952 & 2,01242 \\
53 & 567,4 & 12,34 & 24,18 & 25,01 & 36,91 & 8,0 & 11,52239 & 13,35192 & 1,94179 \\
\hline \multicolumn{7}{c}{ Antes do corte final (CF) } \\
\hline 33 & 395,6 & 16,36 & 29,41 & 30,07 & 40,12 & 20,5 & 15,69916 & 14,72969 & 2,19217 \\
38 & 300,8 & 17,36 & 34,38 & 34,99 & 42,04 & 20,0 & 16,76250 & 19,09266 & 2,83721 \\
43 & 331,3 & 17,48 & 34,84 & 35,54 & 44,54 & 20,3 & 16,79147 & 19,52252 & 2,66712 \\
48 & 299,5 & 19,98 & 38,48 & 39,08 & 47,17 & 20,5 & 19,39549 & 20,71779 & 2,96005 \\
53 & 317,9 & 17,67 & 36,92 & 37,60 & 47,94 & 16,9 & 17,00019 & 21,56854 & 2,95331 \\
\hline
\end{tabular}

Em que $S=$ índice de sítio $(\mathrm{m}) ; D_{\min }=$ diâmetro mínimo $(\mathrm{cm}) ; \bar{d}=$ diâmetro médio aritmético $(\mathrm{cm}) ; d g=$ diâmetro médio quadrático; $D_{\text {máx }}=$ diâmetro máximo $(\mathrm{cm}) ; I=$ idade $(\operatorname{anos})$; " $a$ " = parâmetro de locação; " $b$ " = parâmetro de escala; e " $c$ " = parâmetro de forma.

das simulações para o primeiro desbaste foram realizadas somente nos três sítios intermediários $(38 \mathrm{~m}, 43 \mathrm{~m}$ e $48 \mathrm{~m}$ ), por não haver parcelas disponíveis nos demais sítios para a comparação do desbaste.

Nas simulações de desbaste realizadas para os três índices de sítio, a equação de desbaste, quando aplicada em todas as classes diamétricas, indicava que deveria remover um número de árvores maior do que a média observada. Para contornar esse problema e ter maior controle sobre o número de árvores removidas, foi utilizado o seguinte procedimento:

- Foram removidas todas as árvores das duas menores classes diamétricas.

- Aplicou-se um fator de correção (85\% do número de árvores estimado pela equação de desbaste para ser removido por classe diamétrica), o qual foi definido de acordo com os valores médios removidos observados. Dessa forma, aplicou-se a equação de desbaste para toda a distribuição estimada, removendo todas as árvores estimadas das duas primeiras classes diamétricas; nas demais classes foram removidas apenas $85 \%$ do número de árvores obtidas pela equação de desbaste.
Esse procedimento possibilitou que fossem removidas árvores em todas as classes diamétricas, bem como que fossem removidas todas as menores árvores e que o número de árvores total removido não fosse superior ao que realmente ocorria na prática (de modo que houvesse entre 600 e 700 árvores remanescentes após o primeiro desbaste e entre 250 e 350 árvores remanescentes por hectare, após o segundo desbaste).

As estimativas de $N / h a, G$ e volume, removidos no primeiro desbaste, foram estatisticamente iguais aos dados médios observados pelo teste L\&O (para nível de significância de 1\%) nos três índices de sítio comparados.

Após as simulações do primeiro desbaste, os atributos/características das distribuições diamétricas remanescentes estimadas foram projetados para a idade do segundo desbaste (aproximadamente oito anos), utilizando-se os modelos selecionados anteriormente para a projeção, resumidos na Tabela 3 .

As distribuições estimadas imediatamente antes do segundo desbaste (D2), tanto para o índice de sítio $43 \mathrm{~m}$ quanto para o índice de sítio $48 \mathrm{~m}$ (únicos sítios em que havia dados para comparação), foram aceitas pelo teste K-S (resultado do teste não significativo a $1 \%$ de probabilidade). 
As estimativas obtidas imediatamente antes do segundo desbaste (D2) para o número de árvores foram estatisticamente iguais aos dados médios observados nos índices de sítio 43 e 48 m pelo teste L\&O (para nível de significância de 1\%). Porém, as estimativas de área basal e volume foram estatisticamente diferentes aos dados médios observados nos dois índices de sítio.

Após a projeção dos atributos/características do povoamento e recuperação dos parâmetros da função Weibull na idade do segundo desbaste e estimativa da distribuição teórica dos diâmetros, foi realizada a simulação do segundo desbaste nos cinco índices de sítio. Foram retiradas todas as árvores estimadas para a primeira classe diamétrica, e adotou-se o mesmo fator de correção para a retirada do número de árvores em cada classe, ou seja, eram retiradas apenas $85 \%$ do número de árvores estimadas pela equação de desbaste. As comparações com dados observados foram realizadas de acordo com a disponibilidade destes.

A partir do teste L\&O (para nível de significância de $1 \%$ ), as estimativas obtidas para o $N / h a$ e para a área basal, removidos no D2, foram estatisticamente iguais aos dados médios observados para $S=48 \mathrm{~m}$ e estatisticamente diferentes para o volume. Para o $S=43 \mathrm{~m}$, foram estatisticamente diferentes para as três variáveis.

Após as simulações do segundo desbaste, os atributos das distribuições diamétricas remanescentes estimadas foram projetados para a idade do corte final (em torno dos 20 anos), utilizando-se os modelos resumidos na Tabela 3 .

Com os parâmetros da função Weibull recuperados na idade do corte final (CF) (Tabela 4), foram obtidas as distribuições diamétricas teóricas de cada índice de sítio. Devido à maior amplitude dos diâmetros encontrados no inventário pré-corte, optou-se em utilizar uma amplitude de classe de diâmetro de $5 \mathrm{~cm}$, em vez de $2 \mathrm{~cm}$ como estava sendo utilizado nas estimativas anteriores. As distribuições diamétricas foram estimadas desde o diâmetro mínimo estimado para cada índice de sítio até uma classe diamétrica a mais que o diâmetro máximo estimado. Nogueira (2003) utilizou como limite superior da distribuição teórica dos diâmetros por classes diamétricas a estimativa do diâmetro máximo, acrescentada de duas classes de diâmetro.
A Figura 1 apresenta a distribuição estimada e observada na idade do corte final para os três índices de sítio, em que existiam parcelas disponíveis para comparação. Observa-se, nessa figura, que a distribuição estimada aproxima-se bem das distribuições médias observadas.

As distribuições diamétricas para a idade do corte final foram aceitas pelo teste K-S (resultado do teste não significativo a $1 \%$ de probabilidade) para os índices de sítio $43 \mathrm{~m}, 48 \mathrm{~m}$ e $53 \mathrm{~m}$. As estimativas obtidas para o número de árvores por ha, área basal e volume por classes de DAP não diferiram estatisticamente dos dados médios observados pelo teste L\&O (para nível de significância de $1 \%$ ).

\section{DISCUSSÃO}

\subsection{Sistema de equações para predição de atributos}

O modelo de Feduccia et al. (1979) (9) foi escolhido para estimar o número de árvores sobreviventes nas simulações realizadas, em razão da qualidade do ajuste. Os modelos testados para estimar a área basal utilizados por Santana (2008) apresentaram valores de $\mathrm{R}^{2}{ }_{\text {adj }}$ entre 0,719 e 0,930 , enquanto o modelo selecionado para estimar área basal utilizado por Miguel et al. (2010) apresentou um $\mathrm{R}_{\text {adj }}^{2}$ de 0,940 . O modelo (14), selecionado para este estudo, apresentou precisão compatível com os resultados encontrados pelos citados autores.

Os modelos para estimar o diâmetro mínimo e a variância dos diâmetros foram os que apresentaram as piores estatísticas de ajuste e precisão no sistema de predição de atributos na idade do primeiro desbaste. Esse fato também foi constatado por Santana (2008), que obteve $\mathrm{R}_{\text {adj }}^{2}$ de 0,596 e 0,8806 e $\mathrm{S}_{\mathrm{yx}}$ de $22,3 \%$ e $22,8 \%$ para $D_{\min }$ e $S^{2} d$, respectivamente. Esse autor trabalhou com dados de Eucalyptus grandis na região Nordeste do Paraná. Miguel et al. (2010) conseguiram melhores resultados para o $D_{\min }$, com um $\mathrm{R}_{\text {adj }}^{2}$ de 0,89 e um $\mathrm{S}_{\mathrm{yx}}$ de 9,09\%. Em contrapartida, Scolforo (1990), utilizando vários modelos para estimar o $D_{\text {min }}$ para povoamentos de Pinus caribaea var. hondurensis, localizados na região Sudoeste do Estado de São Paulo, obteve um $\mathrm{R}_{\text {adj }}^{2}$ de 0,9253 e um $\mathrm{S}_{\mathrm{yx}}$ de $1,9675 \mathrm{~cm}$, e para a variância o autor encontrou valores de $\mathrm{R}^{2}{ }_{\text {adj }}$ entre 0,8869 e 0,9858 , superiores aos obtidos pelos outros autores citados e neste trabalho.

Revista Árvore, Viçosa-MG, v.36, n.4, p.719-732, 2012 

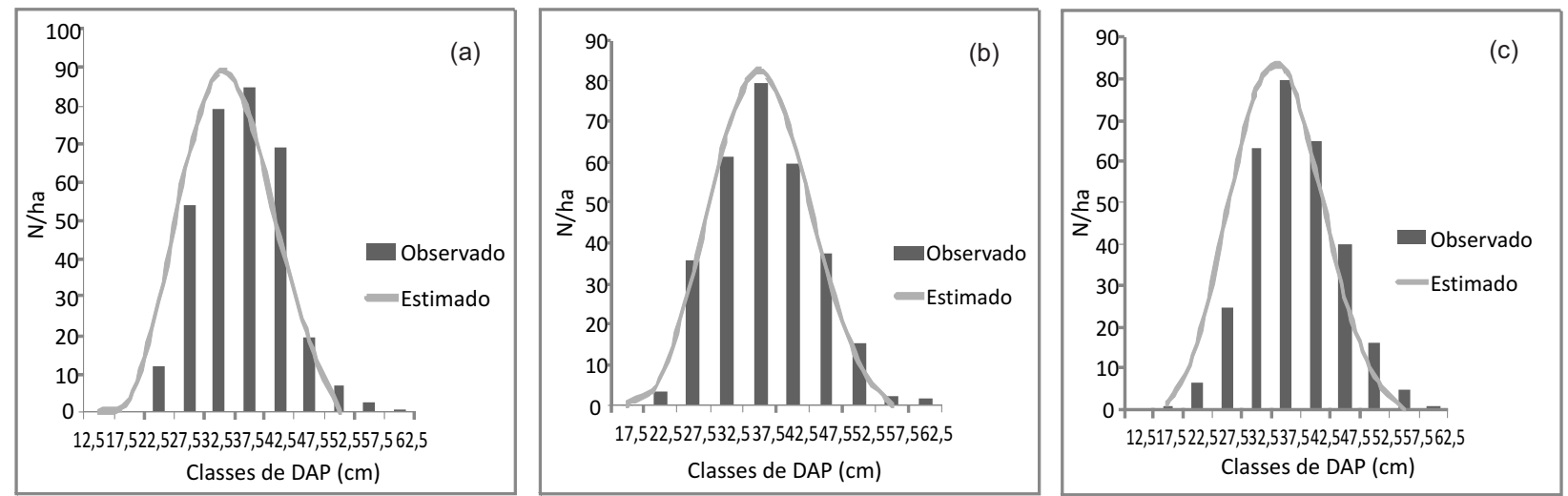

Figura 1 - Distribuição diamétrica estimada e observada na idade de corte final. (a): $S=43 \mathrm{~m}$ (20,3 anos); (b): $S=48$ m (20,5 anos); e (c): $S=53 \mathrm{~m}$ (16,9 anos).

Figure 1 - Diametric distribution estimated and observed in the age of final cut. $(a): S=43 \mathrm{~m}(20,3$ years); $(b): S=$ $48 \mathrm{~m}$ (20,5 years); and (c): $S=53 \mathrm{~m}$ (16,9 years).

Os ajustes dos modelos para estimar $D_{\text {max }}$ foram superiores àqueles obtidos para a variância e o diâmetro mínimo. Wendling (2007) encontrou valores de $\mathrm{R}^{2}{ }_{\text {adj }}$ para o ajuste do Modelo (30) variando de 0,66 a 0,90 para dados de Pinus taeda. Miguel et al. (2010), trabalhando com dados de eucaliptos, obtiveram um $\mathrm{R}^{2}{ }_{\text {adj }}$ de 0,92 para um modelo que estima o $D_{\text {max }}$ em razão da altura dominante.

\subsection{Desbastes}

Para a equação de desbaste, os valores de $\mathrm{R}^{2}{ }_{\text {adj }}$ foram similares no primeiro e no segundo desbaste, porém o $\mathrm{S}_{\mathrm{yx}}$ foi maior no segundo desbaste. Os erros obtidos para estimar o número de árvores removido por classe foram elevados para os dois desbastes ajustados.

Scolforo (1990) utilizou a equação (36) para simular desbastes para dados de Pinus caribaea var. hondurensis e obteve valores de $\mathrm{R}^{2}$ adj que variaram de 0,8538 a 0,9128. Essa equação de desbaste também foi utilizada por Scolforo e Machado (1996), Scolforo et al. (2001) e Acerbi Jr. et al. (2002) para simular vários cenários de desbastes para Pinus sp., gerando boas estimativas para a maioria das simulações.

O procedimento utilizado para controlar o número de árvores removidas no desbaste melhorou as estimativas do número de árvores remanescentes nas classes intermediárias, porém manteve um número de árvores maior nas menores classes. No entanto, se o fator de correção do número de árvores removido não fosse aplicado, o número de árvores remanescentes após o primeiro desbaste seria consideravelmente menor que as médias observadas, o que resultaria em problemas no segundo desbaste e, principalmente, no corte final.

\subsection{Sistema de equações para projeção de atributos}

O modelo de Clutter foi escolhido para a projeção da área basal em função da qualidade do ajuste obtido e também por ser um modelo tradicional para a projeção da área basal. Santana (2008) utilizou o Modelo (38) para projetar a área basal em plantios de Eucalyptus grandis na região Nordeste do Paraná, o qual utiliza uma variável que representa a densidade de árvores por hectare remanescentes do desbaste. Esse autor pressupôs que a presença de tal variável seria interessante para garantir consistência durante simulações de desbastes com intensidades diferentes daquelas observadas.

Os modelos para projetar a variância dos diâmetros apresentaram melhores resultados que os modelos para estimar a variância no sistema de predição. As estatísticas dos ajustes encontradas em Acerbi Jr. et al. (2002) e Santana (2008) para a projeção da variância dos diâmetros apresentaram bons ajustes, como os obtidos neste trabalho.

Nogueira et al. (2005), trabalhando com um sistema para projetar a distribuição diamétrica de eucaliptos submetidos a desbastes, usaram um modelo similar ao modelo (48) para plantios do híbrido E. grandis $\times$ E. urophylla, localizados na região Nordeste do Estado da Bahia. No lugar do diâmetro médio quadrático na idade $2\left(d g_{2}\right)$, esses autores utilizaram o parâmetro de 
escala da função Weibull na idade atual $\left(b_{l}\right)$. Binoti et al. (2010) também modificaram o modelo (48) e usaram o diâmetro médio quadrático na idade 1 ou atual $\left(d g_{1}\right)$ no lugar do diâmetro médio quadrático na idade $2\left(d g_{2}\right)$.

Santana (2008) selecionou o modelo (48) para projetar o $D_{m a x x}$ para dados de eucaliptos. Dos modelos testados pelo autor, esse era o único que apresentava o $D_{\text {maxl }}$ como variável independente. No entanto, neste trabalho o modelo (50) apresentou melhores estatísticas e também utilizava o $D_{\text {máxl }}$ como variável independente.

\section{CONCLUSÃO}

- O diâmetro mínimo e a variância dos diâmetros foram os atributos mais difíceis de serem modelados no sistema de predição de atributos na idade do primeiro desbaste.

- Observaram-se melhores estatísticas de ajuste e precisão nos modelos do sistema de projeção em relação aos modelos do sistema de predição.

- A análise da simulação com a equação de desbaste utilizado por Scolforo (1990) apontou para a necessidade de uma base mais consistente de dados, principalmente nas projeções para o segundo desbaste.

- Na simulação do segundo desbaste, foram encontradas as maiores diferenças entre os valores estimados e observados, podendo ser atribuído a valores médios de poucas parcelas - não representativas ou pela base de dados pequena usada para desenvolver a equação para simular o segundo desbaste.

- O procedimento de controle do número de árvores removido nos desbastes possibilitou que o número estimado de árvores remanescente para o corte final fosse próximo aos valores médios praticados no manejo dos plantios estudados.

- A metodologia empregada possibilita simular o crescimento e a produção em plantios de Eucalyptus grandis ao longo da rotação, gerando resultados adequados, principalmente para o corte final, quando são colhidas as árvores com maiores valores agregados.

\section{REFERÊNCIAS}

ÁLVAREZ, G. J. G. et al. Modelling the effect of the diameter distribution of even-aged Maritime pine stands. Forest Ecology and

Management, v.165, n.1, p.57-65, 2002.
ACERBI JR., F. W. et al. Modelo para prognose do crescimento e da produção e análise econômica de regimes de manejo para Pinus taeda L. Revista Árvore, v.26, n.6, p.699-713, 2002.

ARCE, J. E. Modelagem da estrutura de florestas clonais de Populus deltoides Marsh. através de distribuições diamétricas probabilísticas. Ciência Florestal, v.14, n.1, p.149-164, 2004.

BARRA, O. S. V. et al. Proposta metodológica para o ajuste ótima da distribuição diamétrica Weibull 3p. Floresta, v.34, n.3, p.387-393, 2004.

BINOTI, D. H. B. et al. Uso da função Weibull de três parâmetros em um modelo de distribuição diamétrica para plantios de eucalipto submetidos a desbaste. Revista Árvore, v.34, n.1, p.147-156, 2010.

CAMPoS, J. C. C.; LEITE, H. G. Mensuração florestal: perguntas e respostas. 3.ed. Viçosa, MG: Universidade Federal de Viçosa, 2009. 543p.

CLUTTER, J. L. Compatible growth and yield models for loblolly pine. Forest Science, v.9, n.3, p.354-371, 1963 .

CLUTTER, J. L. et al. Timber management: a quantitative approach. New York: John Wiley \& Sons, 1983.333p.

DIAS, A. N. et al. Emprego de um modelo de crescimento e produção em povoamentos de eucalipto desbastados. Revista Árvore, v.29, n.5, p.731-739, 2005.

FEDUCCIA, D. P. et al. Yields of unthinned loblolly pine plantations on cutover sites in the West Golf region. New Orleans: 1979. 88p. (U. S. Forest Service, Res. Paper SO-148)

LEITE, H. G.; OLIVEIRA, F. H. T. Statistical procedure to test identity between analytical methods. Communications in Soil Science Plant Analysis, v.33, n.7/8, p.1105-1118, 2002.

Revista Árvore, Viçosa-MG, v.36, n.4, p.719-732, 2012 
MALTAMO, M. et al. Comparison of percentile based prediction methods and the Weibull distribution in describing the diameter distribution of heterogeneous Scots pine stands. Forest Ecology and

Management, v.133, p.263-274, 2000.

MIGUEL, E. P. M. et al. Using the weibull function for prognosis of yield by diameter class in Eucalyptus urophylla STANDS. Cerne, v.16, n.1, p.94-104, 2010.

NOGUEIRA, G. S. et al. Determinação da idade técnica de desbaste em plantações de eucalipto utilizando o método dos ingressos percentuais. Scientia Forestalis, n.59, p.51-59, 2001.

NOGUEIRA, G. S. Modelagem do crescimento e da produção de povoamentos de Eucalyptus sp. e de Tectona grandis submetidos a desbaste. 2003. 132f. Tese (Doutorado em Ciência Florestal) - Universidade Federal de Viçosa, Viçosa, MG, 2003.

NOGUEIRA, G. S. et al. Modelo de distribuição diamétrica para povoamentos de Eucalyptus sp. submetidos a desbaste. Revista Árvore, v.29, n.4, p.579-589, 2005.

PIENAAR, L. V. An approximation of basal area growth after thinning based on growth in unthinned plantations. Forest Science, v.25, n.2, p.223-232, 1979.

PIENAAR, L. V.; SHIVER, B. D. Survival functions for site prepared slash pine plantations in the flat woods of Georgia Northern Florida. Southern Journal Forestry, v.5, n.2, p.59-62, 1981.

RETSlafF, F. A. S. Modelagem do crescimento e produção em classes de diâmetro para plantio de Eucalyptus grandis. 2010. 168f. Dissertação (Mestrado em Ciências Florestais) - Universidade Estadual do Centro-Oeste, Irati, 2010.
SANTANA, C. J. O. Simulação do crescimento e da produção em plantações desbastadas de Eucalyptus grandis com diferentes procedimentos de obtenção dos parâmetros da distribuição Weibull. 2008. 100f. Dissertação (Mestrado em Engenharia Florestal) Universidade Federal do Paraná, Curitiba, 2008.

SOARES, T. S. Otimização do uso da madeira em povoamentos de eucalipto. 2002. 49f. Dissertação (Mestrado em Ciência Florestal) - Universidade Federal de Viçosa, Viçosa, MG, 2002.

SCOLFORO, J. R. S. Sistema integrado para predição e análise presente e futura do crescimento e producao, com otimização de remuneração de capitais, para Pinus caribaea var. hondurensis. 1990. 289f. Tese (Doutorado em Ciências Florestais) - Universidade Federal do Paraná, Curitiba, 1990.

SCOLFORO, J. R. S.; MACHADO, S. A. Um sistema de crescimento e produção com simulador de desbaste. Scientia Forestalis, n.50. p.51-64, 1996.

\section{SCOLFORO, J. R. S. Modelagem do} crescimento e da produção de florestas plantadas e nativas. Lavras: Universidade Federal de Viçosa, 1998.

SCOLFORO, J. R. S. et al. Simulação e avaliação econômica de regimes de desbastes e desrama para obter madeira de Pinus taeda livre de nós. Ciência Florestal, v.11, n.1, p.121-139, 2001.

TRINCADO, G. V.; QUEZADA, R. P.; KLAUS, V. G. A comparison of two stand table projection methods for young Eucalyptus nitens (Maiden) plantations in Chile. Forest Ecology and Management, v180, p.443-451, 2003.

WENDLING, W. T. Sistema computacional e modelagem para simulação dinâmica da produção florestal. 2007. 191f. Tese (Doutorado em Engenharia Florestal) Universidade Federal do Paraná, Curitiba, 2007 